\title{
David Koussens, L'épreuve de la neutralité. La laïcité française entre droits et discours
}

Bruxelles, Bruylant, coll. « Droit et Religion », 2015, 211 p.

Jacques Palard

\section{(2) OpenEdition}

\section{Journals}

Édition électronique

URL : http://journals.openedition.org/assr/28265

DOI : $10.4000 /$ assr.28265

ISSN : $1777-5825$

Éditeur

Éditions de l'EHESS

Édition imprimée

Date de publication : 31 décembre 2016

Pagination : 338

ISSN : 0335-5985

Référence électronique

Jacques Palard, «David Koussens, L'épreuve de la neutralité. La laïcité française entre droits et discours ", Archives de sciences sociales des religions [En ligne], 176 | octobre-décembre 2016, mis en ligne le 17 juillet 2017, consulté le 24 septembre 2020. URL : http://journals.openedition.org/assr/ 28265 ; DOl : https://doi.org/10.4000/assr.28265

Ce document a été généré automatiquement le 24 septembre 2020.

(c) Archives de sciences sociales des religions 


\section{David Koussens, L'épreuve de la neutralité. La laïcité française entre droits et discours}

Bruxelles, Bruylant, coll. « Droit et Religion », 2015, 211 p.

Jacques Palard

\section{RÉFÉRENCE}

David Koussens, L'épreuve de la neutralité. La laïcité française entre droits et discours, Bruxelles, Bruylant, coll. « Droit et Religion », 2015, 211 p.

1 L'auteur ne manque pas d'atouts pour étudier les dimensions sociales, politiques et juridiques de la laïcité. À la fois juriste et sociologue, il dirige le Centre d'études du religieux contemporain de l'Université de Sherbrooke, où il occupe également la Chaire de recherche Droit, religion et laïcité. Sa double connaissance de la France et du Québec, où il s'est établi au début des années 2000, lui permet en outre de distiller par petites touches des éléments d'approche comparative, qui vont d'ailleurs bien au-delà des sociétés et des systèmes étatiques français et québécois et qui servent l'objectif de l'ouvrage : présenter une analyse sociologique des aménagements juridiques auxquels la laïcité a donné lieu en France au cours des dernières décennies. Cette analyse se focalise sur les diverses postures adoptées par l'État en matière de régulation de la diversité religieuse afin d'identifier leurs fondements normatifs et d'évaluer leur prégnance sur les modalités juridiques de leur mise en œuvre. Elle se prête constamment au débat scientifique comme en témoignent les références à de nombreux travaux et les 721 notes de bas de page.

2 À la lumière des substrats que constituent les principes fondamentaux et le cadre juridique de la laïcité et de la neutralité de l'État et qui font l'objet des trois premiers chapitres, l'auteur examine les discours et l'établissement de règles de droit auxquels ont donné lieu les principales «mise à l'épreuve» de la neutralité dans la France 
contemporaine (chap. 4 à 6). Il distingue la sécularisation, qui procède d'une individualisation et d'une décléricalisation de la religion, de la laïcisation, qui résulte d'une séparation des normativités religieuses et étatiques, mais il se montre également attentif à l'entrecroisement de ces deux processus et à la multiplicité des aménagements auxquels donne lieu dans une formation sociale la laïcité, délibérément conçue comme plurielle. Deux figures de la laïcité sont ainsi mises en exergue : l'une qui est qualifiée de "nationaliste" et la seconde de "dynamique ». La première, associée à l'histoire singulière des relations entre les Églises et l'État dans une société donnée, est défendue par le courant "néo-républicain", porteur d'une conception substantiviste qui se montre volontiers méfiante à l'égard de toute transcendance et qui est représentée notamment par Henri Pena-Ruiz et Régis Debray. La seconde se traduit plutôt par un mouvement émergent et graduel dont la traduction politique n'est pas dès l'abord conditionnée par l'adoption d'une norme formelle; l'auteur considère que les travaux d'un Jean Baubérot s'inscrivent dans cette perspective, attentive au franchissement de seuils successifs jusqu'à l'adoption éventuelle d'un texte de loi. Diverses dans leurs options, ces conceptions reconnaissent néanmoins les mêmes principes fondamentaux : la liberté de conscience, de pensée et de religion, l'égalité des citoyens et l'autonomie respective de l'État et des religions.

Faisant d'une certaine façon la part qui revient respectivement à l'orthodoxie et à l'orthopraxie, l'auteur voit dans la neutralité à la fois un principe inhérent à la laïcisation dans les démocraties libérales et un indicateur de la réalité politique et juridique de la laïcité. L'examen de certains fondements philosophiques, notamment ceux des Lumières, lui permet d'y déceler une pluralité des formes de la régulation juridique de la diversité religieuse. Une certaine faveur est accordée à John Locke et à sa Lettre sur la tolérance, qui plaide pour la libre expression des convictions religieuses et le libre exercice du culte, alors que la position de Voltaire, dont on sait la virulente critique du catholicisme, est jugée restrictive et que, chez Rousseau, l'appartenance citoyenne prend le pas sur l'appartenance religieuse. Si la pensée du libéralisme politique anglo-saxon, celle en particulier de John Rawls et de Will Kymlicka, accorde une place centrale à la neutralité, c'est d'abord au nom des droits individuels. Koussens souligne que la neutralité de l'État a trait, chez ces penseurs, tout à la fois aux justifications, aux buts et aux effets. Le cadre analytique de l'auteur accorde une grande importance à une double distinction. La première permet de dégager deux idéaux-types de la neutralité : la neutralité confessionnelle, qui conduit à placer les confessions religieuses sur un plan de stricte égalité, et la neutralité référentielle, qui suppose qu'une décision étatique ne se fonde sur aucune conception particulière du bien. La seconde distinction se rapporte aux matériaux analysés : ceux-ci relèvent soit d'une laïcité narrative, lorsqu'il s'agit de rapports de commissions ou de débats parlementaires, soit d'une laïcité juridique, dans le cas des lois et de la jurisprudence.

L'auteur rappelle utilement les principales sources du cadre juridique de la laïcité à la française : les lois scolaires - Ferry et Goblet - des années 1880 et la Loi de Séparation des Églises et de l'État de 1905, dont il est rappelé qu'elle ne se réfère pas expressément à la laïcité ; la Déclaration des droits de l'homme et du citoyen de 1789, en particulier en son article 10 ; les Constitutions de 1846 et de 1958 ainsi que la décision du Conseil d'État du 6 avril 2001, qui élève la laïcité au rang de "principe fondamental reconnu par les lois de la République ». Cette décision fait l'objet d'une intéressante analyse de l'éventuelle constitutionnalité - en fait "difficilement défendable» (p.69) - de ce principe pour la période antérieure à 1946. Les sources internationales et européennes 
complètent ce tableau; si la liberté de religion est garantie par la Charte des droits fondamentaux de l'Union européenne de décembre 2000, l'auteur relève toutefois que la Cour européenne des droits de l'homme n'est pas systématiquement hermétique à la conception française de la laïcité.

Dans son analyse de la neutralité de l'État face à l'expression collective du religieux dans la sphère publique, l'auteur observe que la loi de 1905 n'est pas sans effet sur l'introduction d'un traitement différencié entre les religions: les anciens cultes reconnus bénéficient d'une sorte de prime, du moins jusqu'à l'avis du Conseil d'État d'octobre 1997 qui est venu préciser les conditions d'octroi du statut d'association cultuelle, conditions au premier rang desquelles figure l'exercice du culte comme objet exclusif de la raison d'être. L'examen des travaux de la Commission de réflexion juridique sur les relations des cultes avec les pouvoirs publics, nommée par Nicolas Sarkozy, ministre de l'Intérieur et de l'Aménagement du territoire, et présidée par le professeur de droit Jean-Pierre Machelon, inspire à Koussens une remarque critique : la proposition de création d'un nouveau statut qui bénéficierait d'une « reconnaissance d'utilité publique pour les activités religieuses " lui paraît résulter d'un raisonnement ambivalent en ce qu'il passe outre la non-reconnaissance des cultes inscrite à l'article 2 de la Loi de Séparation. L'auteur se montre également réservé à l'égard de l'extension de la possibilité de garantie des emprunts contractés pour financer la construction d'édifices religieux, et, $a$ fortiori, de l'attribution d'aides directes à cette même fin. Ces propositions n'ont d'ailleurs pas eu de suite: la laïcité juridique est ici demeurée fermée aux arguments de la laïcité narrative. Parmi les autres dossiers qui sont examinés, trois font également l'objet de réserves. Le premier, qui a trait à la politique conduite face aux sectes, permet de montrer la discordance entre la laïcité narrative et la laïcité juridique : à la différence des rapports des deux missions interministérielles successives, la loi de 2001, qui a tendu à renforcer la prévention et la répression des mouvements sectaires, a en effet observé, sur le plan formel, une stricte neutralité confessionnelle. Le second dossier concerne les aumôneries dans les services publics, organisés en application de l'obligation de permettre l'exercice de la liberté de conscience et de religion. L'auteur note que des groupes religieux bénéficient d'une place privilégiée, notamment dans l'armée française; au sein des prisons, l'équilibre est mieux respecté, mais un chemin vers l'égalité entre les cultes reste à parcourir. Enfin, singulièrement au niveau territorial, la collaboration entre les groupes religieux et les pouvoirs publics est sans nul doute vertueuse; toutefois, dans la mesure où « le fait religieux est valorisé alors que la liberté de conscience et de religion protège de façon égale le fait de croire et celui de ne pas croire, ces politiques publiques ne renvoient que faiblement à une position de neutralité confessionnelle » (p.131).

6 Considéré du point de vue du respect de cette neutralité confessionnelle et référentielle, un net clivage paraît s'instaurer entre l'expression collective du religieux et l'expression individuelle, en l'occurrence celle des fonctionnaires de l'État et des citoyens : dans le premier cas, dont relève, on l'a vu, la politique à l'égard des sectes, la laïcité narrative n'a pas trouvé écho dans les normes juridiques ; au contraire, en ce qui concerne l'expression individuelle, l'auteur constate une faible étanchéité entre le discours sur la laïcité et le droit de la laïcité. Le lecteur est fondé à estimer que l'analyse - qui se fait volontiers évaluative - porte ici assez clairement la marque sous-jacente du «modèle » canadien et québécois de gestion politique de la diversité. On sent poindre une prise de position critique à propos de la Charte de la laïcité dans les services publics, largement affichée au cours du printemps 2007 et qui «impose aux usagers 
(donc aux citoyens) certaines obligations et restrictions à l'expression de leurs convictions religieuses que la loi ne leur impose pourtant pas» (p. 37). Il en est de même lorsqu'est évoquée «l'inversion du fardeau de la preuve " relative à la "fidélité " au service public des agents de l'État qui porteraient un signe religieux; c'est aussi le cas à l'égard de ceux des avis du Conseil d'État qui expriment une conception extensive de l'obligation de neutralité des fonctionnaires ou qui font preuve d'une « conception où la sphère publique et l'espace de la vie privée sont très clairement distingués et séparés » (p. 145), à l'encontre, implicitement, de la liberté de conscience et de religion. L'auteur perçoit également une conception maximaliste de la neutralité dans la laïcité narrative telle qu'elle émane du rapport Rossinot de 2006 sur la laïcité dans les services publics ou du rapport du Haut Conseil à l'intégration de 2007 sur le projet de charte de la laïcité dans les services publics. Koussens estime que cette conception maximaliste porte la marque de l'adoption, en 2004, de la loi (qui fait pourtant l'exception) relative à l'expression des convictions religieuses des élèves d'établissements publics d'enseignement du primaire et du secondaire. Jusqu'alors, dans la ligne de l'avis du Conseil d'État du 27 novembre 1989 sur le port du voile à l'école, la laïcité juridique s'était clairement fondée sur la reconnaissance de la liberté de conscience et de religion. La Commission de réflexion sur l'application du principe de laïcité dans la République (dite Commission Stasi) marque une césure : elle a appréhendé la laïcité comme une "valeur républicaine " guidée par une philosophie de l'émancipation au détriment de l'attribution de la primauté à la justice sociale. Dans la foulée, si la loi du 15 mars 2004 a formellement fait preuve de neutralité confessionnelle, elle est malgré tout guidée par un référent idéologique qui n'est pas «neutre»: la laïcité est instrumentalisée au nom de sa valeur civilisationnelle par un État porteur d'un magistère philosophique et d'une conception singulière du bien. Il en est résulté un raidissement de la régulation juridique de la religion, qui n'est pas sans prolongements, au vu des travaux du Haut Conseil, à l'intégration sur l'expression religieuse dans les établissements d'enseignement supérieur. En définitive, «la corrélation qui s'est opérée entre la laïcité narrative et la laïcité juridique a conforté une conception séparatiste des aménagements laïques»(p.178). L'auteur estime toutefois que le caractère partiel, polymorphe et variable de ces aménagements interdit d'adhérer sans autre examen à la thèse d'une « nouvelle laïcité » en contexte français.

7 L'intérêt de l'ouvrage réside en bonne part dans la définition d'une problématique claire, documentée et cohérente qui fournit une lecture originale, parce que distanciée de la laïcité - ou, plutôt, des laïcités - à la française, analysée dans ses discours et dans sa production normative. Cette lecture est opportunément inspirée de la fréquentation et de l'intime connaissance d'un modèle d'action outre-Atlantique dont on trouve la marque dans les travaux de la Commission de consultation sur les pratiques d'accommodements reliés aux différences culturelles qu'ont présidée Gérard Bouchard et Charles Taylor et qui a remis son rapport en mai 2008. Ce modèle joue à la façon d'un révélateur, au sens chimique du terme: il permet en effet de faire apparaître, sous forme d'épreuve, l'image latente des expressions discursives et pratiques de la neutralité, dans ses dimensions à la fois confessionnelle et référentielle. Cette image ne saurait être étrangère à son inscription dans une histoire politique et religieuse qui diffère radicalement de celle du Québec et du Canada. C'est dire que les préférences politiques, qui sont aussi le produit de références culturelles, apparaissent avec plus de netteté sous la plume d'un observateur avisé qui sait se tenir à bonne distance. 